\title{
From evidence to implementation: Introducing neonatal simulation to a tertiary neonatal centre in the $\mathrm{UK}^{*}$
}

\author{
Alok Sharma \\ Neonatal Unit, Princess Anne Hospital, Southampton, UK \\ Email: aloksharma@nhs.net \\ Received 3 January 2013; revised 8 February 2013; accepted 18 February 2013
}

\begin{abstract}
Simulation training in medical education allows multidisciplinary teams to train in a realistic clinical environment. It helps reduce clinical errors and addresses patient safety issues. Multiple areas can be covered including procedural skills training, communication, teamwork and human factors training. We present a "model of simulation" in education, which is based on Royal College of Paediatrics and Child Health United Kingdom general paediatric curriculum. Simulation complements the existing neonatal education programme both for doctors and nurses. It has the long-term purpose of achieving higher standards in neonatal care through the training of staff in a structured, multidisciplinary environment. Its quality and impact are subject to feedback from the participants. This has helped in its evolution and development as a multidisciplinary programme.
\end{abstract}

Keywords: Simulation; Education; Neonatal

\section{INTRODUCTION}

"The unfamiliar was familiar because it had been encountered many times before.”

These are the words of Liam Donaldson endorsing the need for simulation in training the medical workforce in the United Kingdom as part of the Chief Medical Officers report in 2008 [1].

Simulation in medical education has been recognised and adopted by a variety of specialties in response to healthcare and safety issues worldwide. It is estimated that in the United States medical errors are the 8th leading cause of death at a cost of $\$ 29$ billion dollars annually [2]. The National Patient Safety Agency receives approximately 300 serious patient safety inci-

\footnotetext{
"Conflict of interest: There are no conflicts of interests involved in the writing of this article. The author is employed as a neonatal consultant and no funding was solicited for this work. There are no other sources of funding and or affiliations of the author with any outside companies of any sort in the public, commercial or the not-for-profits-sector.
}

dents reported by staff in the National Health Services United Kingdom every week [3].

Simulation allows multidisciplinary teams to train in an interactive environment facing real clinical scenarios. It helps to develop team work and communication skills across specialties, as well as allow training in rare and complex scenarios [3]. This is best exemplified in neonatology where obstetricians, midwives, neonatal nurses, neonatal nurse practitioners and doctors work together to bring about the best outcomes for the baby, often in highly stressful situations. Simulation provides a safe environment where rare high-risk events are reproducible. Changes in the structure of medical training in the United Kingdom have resulted in a reduction of junior doctors' hours and a concern regarding the ability to obtain key competencies when addressing critical events. Indeed, the need for simulation as a model for medical education in paediatrics has been identified in previous eras such as the Calman years of paediatric training $[4,5]$.

The Royal College of Paediatrics has not to date published any guidelines or a curriculum under which simulation can be implemented either in paediatrics or neonatology. This has not prevented individual centres from embracing simulation as a modality of education for their teams in paediatrics, paediatric intensive care and neonatal intensive care through the use of existing $\mathrm{RCPCH}$ curriculum in the UK.

Significant work has been performed by Fawke et al. in Leicester, where a high fidelity point of care neonatal simulation programme has been established and operated for over 3 years. This programme has been operated in the form of fortnightly sessions that are run by a multidisciplinary team replicating the working environment [6]. Similar programmes are being run in other neonatal centres, and a national neonatal simulation training group called NeoSim UK has been established. The group shares ideas and expertise with regards to the development of simulation as a modality for neonatal education.

Simulation suites have been developed in centres around the UK. This allows for simulation training outside the working environment but the introduction of an 
effective "point of care high fidelity simulation programme" remains a challenging prospect that may take years to achieve [6].

\section{SIMULATION IN NEONATAL EDUCATION}

Neonatology involves professionals from multiple different specialties, such as midwifery, nursing, paediatrics, and tertiary specialities, working together. The education and training of these professionals generally occurs separately. Neonatal simulation allows these professionals to train together and work as a team, as they would in the clinical environment.

Traditionally audit and research, e-learning, seminars, lectures, external training courses, reflective practice and self-directed learning have been used for delivery of the neonatal and paediatric curriculum in the United Kingdom. Procedural skills are demonstrated, observed and practiced [7,8]. Programmes such as the Neonatal Life Support course run by the Resuscitation Council use low-fidelity simulation for the acquisition of neonatal resuscitation skills. A high fidelity programme helps complement existing models for the delivery of education. Simulation training can improve outcomes in the clinical setting. Healthcare professionals training with a PROMPT birthing trainer simulator have reported a decrease in the use of harmful delivery interventions for Shoulder Dystocia. This has resulted in better clinical outcomes for neonates with a reduction in neonatal injuries after birth through Shoulder Dystocia after such training was introduced [9]. In a simulation-based model, learning occurs via active participation rather than through a review of literature or passive observation alone. Simulation in neonatology allows exposure to high-risk events without putting neonates at risk while allowing individuals to develop the cognitive, technical and behavioural skills necessary to address such events when they actually occur. It also helps develop teamwork, communication and prioritisation skills in stressful situations [10]. A number of trainees experience anxiety at the start of their neonatal training and when they assume more middle grade responsibilities. Simulation training performed prior to real patient exposure may be helpful in such situations. Simulation courses addressing such needs have been run in an established simulation programme in Bristol [11].

\section{INTRODUCING NEONATAL SIMULATION “A NEONATAL MODEL”}

In keeping with available evidence and after literature review the potential for the introduction of simulation training in neonatology in Southampton was discussed. A faculty was convened comprising a neonatal con- sultant and designated specialist registrar who was responsible for identifying target training groups, developing a curriculum, utilizing existing resources and designing scenarios. Two specialist registrars and a nurse lead were trained at the Leicester Neonatal Simulation Instrucor's Course and the Southampton simulation centre.

The simulation suite and a designated isolation room on the neonatal unit were identified as venues for simulation sessions, and all sessions have been carried out at in these set areas. Scenarios were prepared using the RCPCH curriculum initially addressing common problems of the neonatal airway and breathing and progressing to more complex areas [7]. Targeted pilot sessions were carried out covering year 1 - 3 trainees on the neonatal unit and year 4 - 8 trainees on a regional training day. Procedural skills' training was also piloted. Sessions to familiarise the nurses with the mannequin were run on the neonatal unit.

Feedback collated from these sessions was used to design a programme involving the year 1 - 8 trainees, neonatal nurse practitioners and nurses on the neonatal unit. A pool of scenarios was prepared. The scenarios were prepared in the context of clinical cases with a predicted clinical course and structured to cover various systems (Figure 1).

High fidelity scenarios were run on the Sim Baby (Laerdal). This mannequin provided the trainees with cues to aid in decision making while the mannequin was programmed to react in realistic ways to the trainees' interventions. Trainees were challenged to apply the correct intervention to improve the condition of a simulated patient in an environment that attempted to imitate our neonatal unit. Low fidelity scenarios involved using the neonatal Sim Anne (Laerdal). Preterm scenarios involved use of the Simulaid Micropremmie. Low fidelity scenarios were based on communication, organisation or the ability to approach a particular neonatal problem. This scenario's aimed to address crisis resource management [5].

Each scenario was run over 60 minutes, with an introduction followed by the simulation sequence and

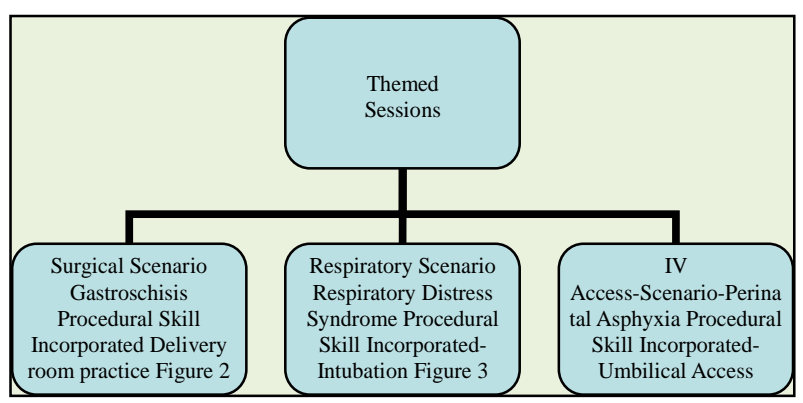

Figure 1. Themed neonatal simulation. 
feedback. The introduction and scenario were run for 20 minutes followed by debriefing and teaching about points raised in the scenario in the remaining time. Feedback was obtained on the sessions and scenarios.

Monthly sessions were planned in advance and carried out as themed sessions covering the neonatal airway, access, respiratory, circulatory, and surgical problems. Sessions included procedural skills training in addition to simulation training on the day. Simulations were tailored to allow participants to enact their roles and assessments as they would on the neonatal unit.

\section{THE SOUTHAMPTON NEONATAL SIMULATION PROGRAMME-“THE RESULTS OF A PILOT”}

Simulation was piloted on the neonatal unit at Southampton in December 2010. It was run without any funding, and individuals using their own initiative and time did significant work.

A total of 14 sessions were run between December 2010 and December 2011, including 2 regional study days. Health professionals including year 1 - 8 trainees, neonatal nurse practitioners, and nurses participated in the program. Feedback was obtained as in Table 1.

Certain areas of risk were identified on the neonatal unit, including endotracheal tube fixation and leaking umbilical lines. These issues were addressed con-currently during scenarios and procedural skills training.

The feedback provided also identified areas for improvement. Realism was not always achieved during all of the scenarios because of the absence of a neonatal simulation mannequin. There was difficulty organising the availability and presence of nursing staff, which resulted in a compromised degree of realism and fidelity for certain scenarios. The absence of a multidisciplinary

Table 1. 85 feedback forms answered the following questions in 2011.

\footnotetext{
Do you think neonatal simulation should be part of training?

92\% agreed, 8\% no response.

Was it relevant to my training?

82\% strongly agreed, $17 \%$ agreed, 1\% disagreed.

Was it relevant to my clinical practice?

80\% strongly agreed, 14\% agreed, 6\% no response.

Was multi-professional feedback relevant and useful?

70\% strongly agreed, 22\% agreed, 8\% no response.

Overall the neonatal simulation education session was of high quality?

78\% agreed, $21 \%$ agreed, $1 \%$ neutral.
}

curriculum and varied learning objectives between different professionals meant that all learning needs might not have been specifically addressed.

Feedback from our programme was collated to produce a business case for a high fidelity neonatal mannequin. The data and the business case was presented to local deanery officials resulting in the purchase of 2 high fidelity simulation mannequins, specifically neonatal mannequins to perform procedures such as line insertion and lumbar puncture.

A dedicated simulation suite, with the availability of high and low fidelity neonatal simulation mannequins (Figures $\mathbf{2}$ and $\mathbf{3}$ ) and appropriately trained faculty, has allowed for the development of a point of care simulation programme.

Existing medical and nursing curriculum is being integrated through curriculum mapping to ensure that training is beneficial to the multidisciplinary groups participating in the sessions. Patient safety issues raised through local clinical governance procedures are also being contemporaneously addressed through the simulation sessions run.

\section{CHALLENGES OF STARTING A MULTIDISCIPLINARY NEONATAL SIMULATION PROGRAMME}

It is expected that a number of neonatal units would like to embrace simulation as a training modality to complement existing models of education for staff. The first step in any programme is identifying existing resources in terms of faculty and simulation infrastructure. There are simulation instructor courses run in Leicester Royal Infirmary and Bristol Education Centre in the United Kingdom catering to nurses and doctors [6,11]. Establishing faculty among the different multidisciplinary group's nurses, doctors and midwifery broadens the ability to identify the learning needs of different groups as well adapting different curriculum types.

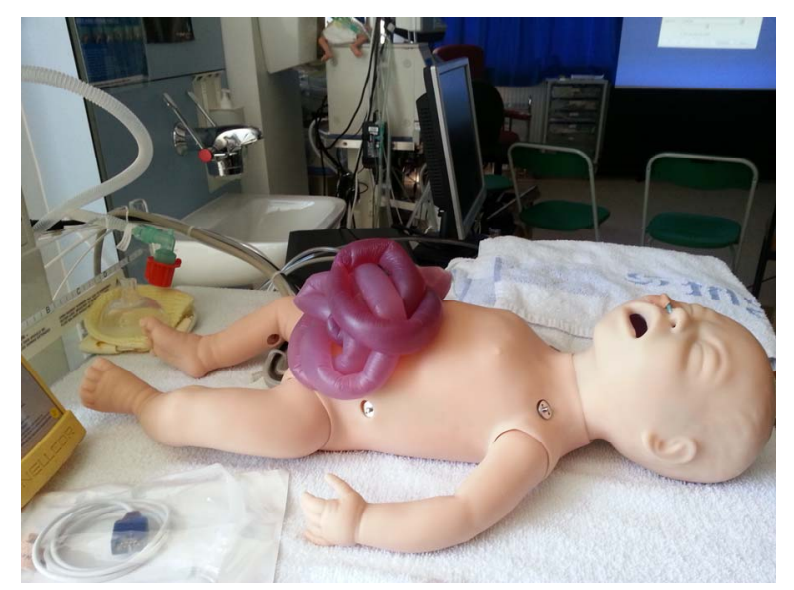

Figure 2. Gastroschisis model high fidelity mannequin. 


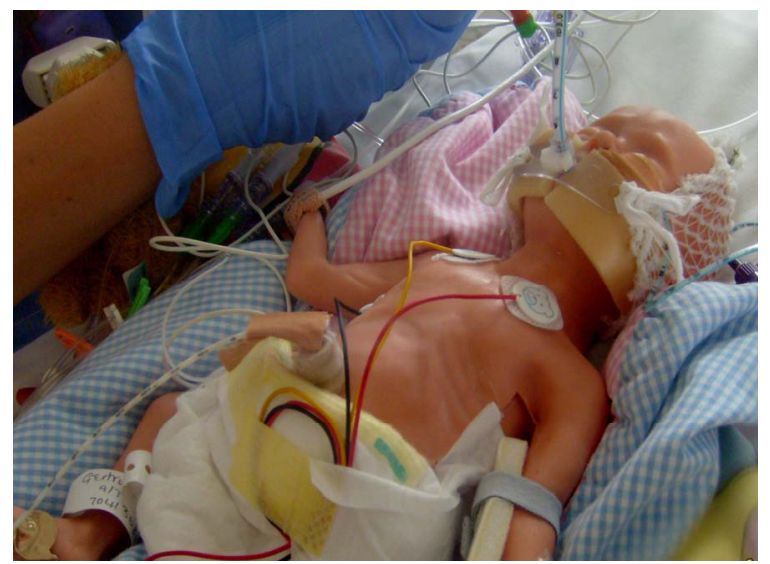

Figure 3. Respiratory distress syndrome preterm low fidelity mannequin.

An existing curriculum to draw upon for scenarios along with enthusiastic individuals helps with the next stage. A review of incident reports and serious untoward incidents can help produce scenarios that address issues of risk locally. Simulation training requires dedicated time, and staff needs to be fully involved and familiar with the mannequins and simulation environment. In our experience, a period of prior familiarisation followed by dedicated training days for doctors and nurses, as opposed to work-based learning, ensures better attendance and achievement of desired learning outcomes.

Identifying a suitable environment is paramount. A setting on the neonatal unit will require technology that is compatible with operating the mannequin and should ideally also not disturb day-to-day practice on the neonatal unit

Finally, having a high fidelity simulation mannequin is ideal, but not having one should not be a deterrent if other mannequins are available for use. Low fidelity mannequins can be used for team work training and crisis resource management.

\section{Fidelity and Your Programme}

There is an emphasis on the use of high fidelity mannequins and the recreation of the exact clinical environment so that individuals obtain the highest degree of learning. Issenberg et al. have identified some of these principles. Simulator validity and providing effective feedback are very important tenets of this emphasis [12]. A stumbling block is the complexity of neonatal assessment and its duplication.

Achieving high fidelity in an established simulation programme takes years of hard work, faculty training, and practice. In the formative stages of developing a programme, a compromise in the degree of fidelity is inevitable. It must also be remembered that facilitators are not born with but learn debriefing skills. We must be careful to see that we are achieving desired learning out- comes despite this for staff involved in the training.

This model demonstrates that while aspiring to achieve long term goals of delivering multidisciplinary training through high fidelity simulation, we can encourage learning through modelling scenarios with lesser fidelity but with defined learning objectives. An important aspect of setting up appropriate simulation training scenario's is designing them so that the degree of fidelity allows participating individuals to come to some desired learning outcomes without feeling cheated by the mannequin and scenario. Debriefing is a critical aspect of this process, and feedback about the scenarios is a major driver for improvement. Designing a few scenarios that can be practised and piloted with feedback has worked well in our set up. We provide 2 sample scenarios below, in Tables 2 and 3.

\section{MULTIDISCIPLINARY SIMULATION TRAINING}

Having curriculum-based scenarios that are credible, observed clinically, and reproducible in your structured environment will be much more successful in achieving a desired learning outcome [11,12]. We run a scenario based on the approach to delivery of the extreme preterm neonate on a low fidelity micropremmie doll (Table 2). This scenario focuses on teamwork and preparation, including early management and temperature control as learning objectives. Using the high fidelity neonatal mannequin, although it would allow the added advantage of intubation, would be less realistic due to its size. Fitting it into our plastic bags would be a major challenge. Effective communication between the doctor and the nursing team is the objective here, not intubation.

On the other hand, situations involving decision making based on clinical assessment and recognition of dynamic changes are better performed on a high fidelity simulator. Examples of this in our programme are the resuscitation of a neonate with respiratory distress (Table 3). The neonatal sim baby allows demonstration of cyanosis, desaturation and reduced air entry. Airway management including intubation are learning objectives of this scenario. While intubation is a medical learning outcome, preparation and supporting of a junior doctor in the process would be learning outcomes for our nursing colleagues.

Learning objectives for different professionals might be the same or different using the same scenario. The difficulty of the scenario and the competency of the individuals involved need to be kept in mind [11]. A scenario on accidental extubation covers the ability of a nursing colleague to recognise respiratory deterioration, initiate management and call for help. The same scenario then covers the ability of a doctor to manage the airway and breathing. A common clinical learning objective would 
Table 2. Team approach to delivery of an extremely premature baby.

Mannequin Simulaid Micropremmie
Fidelity Low
Team Registrar/SHO/Nurse-Venue NNU Side Room

History-You are called to a category 2 LSCS of a breech male 26 week baby at 0200 . The mother has been admitted for recurrent APH and was being observed but the foetal movements were reduced and CTG is borderline. Steroids were given 24 hours previously.

1) Prepare your team-Ask for an experienced senior nurse and inform consultant. Prepare your team with roles-designate specific roles for airway, access and monitoring.

Expected actions: $\quad$ bag/transwarmer, saturation probe, fixation equipment (Hat, forceps), and surfactant administration equipment.

3) Check resuscitaire-blender, FIO2 air to start, Pressures Max 20 to start titrated up if necessary, check that the necessary equipment and temperature probe are available.

Rationale:

Expected actions:

SIM:

Actions:

SIM:

Actions:
Preparation/Communication Is Essential.

The baby is delivered.

Put the baby in the plastic bag, nurse attaches saturation probe to the right forehand, and assesses breathing, heart rate, colour, and tone. Nurse to attach temperature probe. Team assumes designated roles. Call for senior help designating a person of the team to call the consultant.

Heart rate—60 beats/min. Ineffective breathing efforts, floppy, cyanosed (Facilitator prompts)

When no respiratory effort is observed, based on competency choose to intubate or provide IPPV and reassess. Airway manoeuvres might be necessary. (They are possible on this mannequin)

If the saturation probe is applied the monitor will be used to duplicate heart rate and oxygen saturation as per intervention.

Airway and breathing managed as per competency. Nurse is to prepare surfactant if intubated and scenario ends.

Learning points: This scenario is assessing the team approach to the extreme newborn and the ability of the NNU team to prepare for a delivery of the extreme preterm in which time is available. The medical management of the baby is not the focus. Identifying roles as designated by a team leader and preparation are essential along with the specific instruction of calling for a consultant.

Table 3. Airway management of a neonate with respiratory distress.

\section{Mannequin Laerdal Sim Baby \\ Fidelity High \\ Team Consultant/Registrar/SHO/Nurse Venue NNU Side Room \\ You are called to the Elective LSCS of a woman who is 36 weeks gestation with transverse lie. She has not been in labour for long and the abnormal position of the baby has prompted need for the LSCS.}

Expected actions: $\quad$ Check equipment, and ask for nursing help.

Rationale:

\section{Preparation is essential.}

SIM:

Baby delivered-Blue, heart rate $>100$ beats/min, intermittent, inadequate respiratory effort.

Expected actions:

Dry, remove wet towel, and stimulate, and Assess breathing, heart rate, colour, tone.

SIM:

As above with intermittent apnoea. No improvement with stimulation. Chest has crepitations, heart rate falling to 60 beats/min.

When no respiratory effort is observed, commence inflation breaths or intubate while looking for chest movement or improvement in colour.

SIM: $\quad$ Heart rate dropped to 60 beats/min, blue; if saturation probe applied saturation will be $50 \%$, with no chest movement. Manage airway as per competency-Reposition/2 person technique to IPPV/effective mask seal/Suction under direct

Actions: vision if concerns regarding airway secretions. Watch for chest wall movement. Prepare for intubation. Call for senior help.

Poor respiratory effort with IPPV. Heart rate is 100 beats/min Saturations remain low at $60 \%$. Poor air entry on the mannequin.

SIM:

Attempt Intubation. If unsuccessful manage airway as above and call for help.

Actions:

Heart rate and saturations improve with appropriate management of airway and breathing. If the ET tube goes down

SIM: the right main bronchus, saturations will remain low thus prompting reassessment.

Actions: $\quad$ ET needs appropriate fixation and to be pulled back if necessary.

Learning points: This baby has respiratory distress and needs appropriate airway/breathing support. This scenario assesses the ability of the team to manage airway and breathing through intubation or appropriate airway manoeuvres until senior help arrives. 
be the diagnosis of a blocked or displaced endotracheal tube; however, issues with teamwork, behavior or knowledge of one's own limitations might emerge as individual learning outcomes.

Currently, there is no defined curriculum for multidisciplinary training in neonatology in the United Kingdom. This means that the learning objectives defined for scenarios based on the RCPCH curriculum [7] might not necessarily meet the needs of nursing and midwifery colleagues. As educators, we must not presume learning objectives for colleagues in other fields. The focus should be on how we can adapt existing curriculums to meet competencies and learning objectives for all participating individuals. Involving senior educators from both of these teams is an essential aspect of the process. Scenario development should cover multidisciplinary teamwork training. A previous study highlights the importance of teamwork training with respect to quality of neonatal care [13]. The PROMPT model implemented by Draycott et al. shows how such training can make a difference to neonatal outcomes. A syllabus has been produced to address neonatal competencies for neonatal nurses which is endorsed by the Royal College of Nursing and the British Association of Perinatal Medicine. This document is being used for curriculum integration in our programme [14].

\section{STANDARDS FOR NEONATAL SIMULATION TRAINING}

Established programmes of international repute include the Centre for advanced pediatric and perinatal education based at Stanford University. Simulation is delivered as courses covering the neonatal, paediatric and obstetric domains [10]. The Leicester neonatal simulation programme runs fortnightly sessions using neonatal, delivery suite and transport scenarios and has been running for years. Our programme being in its formative stages differs from other neonatal simulation programmes in being a modular multidisciplinary programme covering neonatal doctors, nurses, and nurse practitioners at present. We run monthly modules covering the neonatal airway, access, respiratory system, circulation, surgical and nervous system incorporating sessions for procedural skills (Figure 1). Scenarios also incorporate issues raised locally through clinical governance.

Neonatal simulation training is not used for formal accreditation of competencies in our programme. The approach is to use it as complementary to other methods of training, not for assessment. The focus is on human factors training, multidisciplinary team work and communication and discussing medical errors. At present models, task trainers and neonatal high fidelity patient simulators may be useful for assessment of clinical practice in neonatology [9-12], but there needs to be a specialty specific formal evaluation of validity, reliability, and feasibility in their use for formal accreditation of competencies and assessment. At this point in time an effective model for assessment using the above is lacking.

With different programs having different methods of using and implementing simulation in neonatology maintaining a standard to ensure participants are achieving learning is important. All our scenarios are curriculum based. As a minimum in order to ensure quality of sessions we try to ensure the presence of both nurses and doctors to participate in their roles, plausible scenarios and 2 or more facilitators to run each session. Feedback is taken for all sessions. All of our nurses are expected to rotate through the sessions over 18 months while all junior doctors rotate every 6 months. An audit of sessions arranged in 2012 showed that there were 2 facilitators for $100 \%$ of sessions and $82 \%$ of sessions were multidisciplinary sessions. Obtaining feedback is an important to help improve upon the sessions and running of the programme. Having experienced facilitators is very important. At present all instructors in paediatric simulation in Southampton are trained through established simulation programmes such as those run in Leicester by Fawke et al. [6] and Bristol [11] in the United Kingdom. There are currently no formal accreditation programmes for certification of medical simulation instructor training in the United Kingdom; however the society for simulation in healthcare has recently piloted the Certified Healthcare Simulation Educator (CHSE) programme. This consists of an exam. The standards were developed through a consensus approach with representatives from many countries and societies all experts in the field of simulation [15]. At present an area of active research in our programme is feedback from different participating multidisciplinary groups. Feedback is being collated separately from neonatal nurses, trainee's year 1 - 8, neonatal nurse practitioners and neonatal consultants who are debriefed together to try and assess whether educational quality of the simulation sessions meets needs of different groups.

\section{CONCLUSION}

Introducing simulation in any form requires significant resources, time, motivation and effort to change the learning mindset of individuals who have not participated in this type of training before. Neonatal simulation allows multidisciplinary teams to develop skills on a safe acceptable substitute for the patient while aspiring for excellence in neonatal care. We propose simulation as an important adjunct to other methods in neonatal education and provide a model for the same. This model is reproducible, curriculum based with the objectives of trying to 
achieve higher standards in multidisciplinary team care.

\section{ACKNOWLEDGEMENTS}

I would like to thank the following individuals who have been involved with the inception of the pilot programme Dr. S Francis, Dr. Helen Fielder, Dr. R Ungureanu, and Dr. David Burge.

\section{REFERENCES}

[1] Donaldson, L. (2009) Safer medical practice: Machines, manikins and polo mints, 150 years of the annual report of the chief medical officer: On the state of the public health 2008. Department of Health, London, 49-55.

[2] Kohn, L.T., Donaldson, M.S. and Corrigan, J.M. (1999) To err is human: Building a safer health system. National Academy Press, Washington DC.

[3] National Patient Safety Agency-NPSA (2012) Patient safety and simulation: Using learning from national review of serious incidents (January 2010). http://www.nrls.npsa.nhs.uk/resources/type/guidance/?ent ryid45 $=74297$

[4] Templeton, J. (2010) A review of the impact of European working time directive on the quality of training. Medical Education.

http://www.mee.nhs.uk/PDF/14274\%20Bookmark\%20W eb\%20Version.pdf

[5] Stewart, D. (2003) Medical training in the UK. Archives of Disease Childhood, 88, 655-658. doi:10.1136/adc.88.8.655

[6] Fawke, J., Cusack, J. and Sood, A. (2011) The Leicester neonatal high fidelity simulation programme. Archives of Disease in Childhood_Fetal and Neonatal Edition, 96, Article ID: Fa36.

[7] Royal College of Paediatric and Child Health-RCPCH (2012) Curriculum for paediatric training neonatal medicine level 1, 2 and 3 training (Sept. 2010). RCPCH Website.

http://www.rcpch.ac.uk/system/files/protected/page/2010

\section{\%20Neonatology\%20curriculum.pdf}

[8] McGraw, M.E. (2009) Delivery of the paediatric curriculum of the Royal College of Paediatrics and Child Health (RCPCH). Archives of Disease in Childhood, 94, 254-257. doi:10.1136/adc.2008.137679

[9] Draycott, T.J., Crofts, J.F., Ash, J.P., et al. (2008) Improving neonatal outcome through practical shoulder dysntocia training. Obstetrics \& Gynecology, 112, 14-20. doi:10.1097/AOG.0b013e31817bbc61

[10] Yaeger, K.A., Halamek, L.P., Coyle, M., Murphy, A., Anderson, J., Boyle, K., Braccia, K., McAuley, J., Sandre, G.D. and Smith, B. (2004) High-fidelity simulation-based training in neonatal nursing. Advances in Neonatal Care, 4, 326-331. doi:10.1016/j.adnc.2004.09.009

[11] Grant, D.J. and Marriage, S.C. (2012) Training using medical simulation. Archives of Disease in Childhood, 97, 255-259. doi:10.1136/archdischild-2011-300592

[12] Issenberg, S.B., McGaghie, W.C., Petrusa, E.R., Lee Gordon, D. and Scalese, R.J. (2005) Features and uses of high-fidelity medical simulations that lead to effective learning: A BEME systematic review. Medical Teacher, 27, 10-28. doi:10.1080/01421590500046924

[13] Thomas, E.J., Sexton, J.B., Lasky, R.E., Helmreich, R.L., Crandell, D.S. and Tyson, J. (2006) Teamwork and quality during neonatal care in the delivery room. Journal of Perinatology, 6, 163-169. doi:10.1038/sj.jp.7211451

[14] British Association of Perinatal Medicine-BAPM (2012) Matching knowledge and skills for qualified in speciality (QIS). Neonatal nurses: A core syllabus for clinical competency April 2012.

http://www.bapm.org/publications/documents/training/M atching\%20Knowledge\%20and\%20Skills\%20for\%20QIS \%20Neonatal\%20nurses\%20-\%20a\%20core\%20syllabus \%20for\%20cllinical\%20competency.pdf

[15] Society of Simulation in Healthcare (SSH) (2013) Certified healthcare simulation educator. http://ssih.org/uploads/static_pages/PDFs/Certification/C HSE_Handbook.pdf 